
allemande

46-2 | 2014

Intellectuels et politique en Allemagne

\title{
Vom Krieg der Großmächte zur Katastrophe Europas
}

Gerd Krumeich

\section{(2) OpenEdition}

1 Journals

Édition électronique

URL : https://journals.openedition.org/allemagne/1166

DOI : 10.4000/allemagne. 1166

ISSN : 2605-7913

Éditeur

Société d'études allemandes

Édition imprimée

Date de publication : 30 décembre 2014

Pagination : 411-430

ISSN : 0035-0974

\section{Référence électronique}

Gerd Krumeich, „Vom Krieg der Großmächte zur Katastrophe Europas“, Revue d'Allemagne et des pays de langue allemande [Online], 46-2 | 2014, Online erschienen am: 29 Juli 2019, abgerufen am 19 Mai 2021. URL: http://journals.openedition.org/allemagne/1166; DOI: https://doi.org/10.4000/allemagne. 1166 


\section{Vom Krieg der Großmächte zur Katastrophe Europas}

\section{- Gerd Krumeich*}

\section{Vorstellungen vom Krieg}

Nichts ist in den Darstellungen zum Beginn des Ersten Weltkrieges geläufiger als die Feststellung, dass die Staatsmänner von damals mit ungeheurer Leichtfertigkeit einen Krieg vom Zaun gebrochen hätten, der dann das Alte Europa zerstört und die „Urkatastrophe“ des 20. Jahrhunderts gewesen sei. Am nachhaltigsten wird diese Vorstellung aktuell von der Metapher der „Schlafwandler“ genährt. Das gleichnamige Werk des in England lehrenden australischen Historikers Christopher Clark hat es so suggestiv eingesetzt und so eloquent beschrieben, dass er einen auf dem Markt der historischen Fachliteratur noch nie dagewesenen Erfolg verbuchen konnte. Allein in Deutschland wurden innerhalb nicht einmal eines Jahres 200.000 Exemplare in 18 Auflagen verkauft ${ }^{(1)}$ ! Clarks Bild der schlafwandlerisch handelnden Diplomaten der Zeit vor 1914 gemahnt auf den ersten Blick an ein anderes und jahrzehntelange maßgebendes Diktum, nämlich dass die militärisch und politisch Verantwortlichen der europäischen Nationen in diesen Krieg „hineingeschlittert seien“, wie es das ebenfalls vielzitierte Wort des britischen Politikers David Lloyd George von 1933 wollte:

„Nicht einmal die scharfsinnigsten und weitestblickenden Staatsmänner haben selbst im Frühsommer 1914 vorausgesehen, dass mit dem Herbst die Völker der Welt in den schrecklichsten Zusammenstoß verstrickt werden würden, den es in der der Geschichte der Menschheit jemals gegeben hat... [...] Ohne die geringste Spur von Angst oder Bestürzung rutschten („slithered“) die Völker über den Rand in den siedenden Kessel des Krieges“(2).

Doch Clarks Bild der Schlafwandler zielt weiter als das „Hineinschlittern“ bei Lloyd George: für Clark verhielten sich die politisch Verantwortlichen sehr zielgerichtet und gradlinig. Aber ihnen habe es an jeglicher Empathie, jeglichem Verständnis für den

* Professor emeritus der Heinrich Heine Universität Düsseldorf.

1 Christopher Clark, Die Schlafwandler. Wie Europa in den Ersten Weltkrieg zog, München, 2013, 13. Auflage, März 2014.

2 Zit. nach: David Lloyd George, Mein Anteil am Weltkrieg (War Memoirs), Bd. 1, Berlin, 1933, S. 41. 
Gegner bzw. den potentiellen Kriegsgegner gefehlt. Sie seien allein am eng gefassten Eigeninteresse orientiert gewesen und hätten sich deshalb mit tödlicher Konsequenz folgerichtig verhalten, eben wie ein Schlafwandler, der sich auch nicht vom Gitter seines Balkons abhalten lässt, den einmal eingeschlagenen Weg fortzusetzen und der deshalb notwendigerweise in den Tod stürzt. Dieses Bild von Christopher Clark ist enorm verführerisch, es lässt eine ungeheure Dynamik des Verhaltens ahnen, es entlastet gleichzeitig ungemein, denn schließlich kann man einem Schlafwandler sein Verhalten ja nicht zum Vorwurf machen. Letztlich bleibt alles ein dunkles Fatum, welches die Menschheit in die Katastrophe von 1914 geworfen hat. Es sei allerdings einschränkend hinzugefügt, dass sich bei Clark in der konkreten Darstellung der Ereignisse die Verantwortlichkeiten implizit und auf der Ebene von Nebenbemerkungen und Schwerpunktsetzungen doch ein wenig verschieben. Es scheint, als ob der deutsche Michel eher nur vor sich hinträumte, ja: ein veritabler Traumtänzer war, wohingegen die Russen und die Franzosen, auch die Serben, eine die Krise verschärfende Dynamik in Gang setzten und bis zum bitteren Ende immer weiter verschärften.

Eine wichtige Frage wird allerdings mit der „Schlafwandler“-Metaphorik überhaupt nicht thematisiert und ist auch ohnehin in der Literatur zur Entstehung des Ersten Weltkriegs bislang nur höchst selten gestellt worden, nämlich: Haben die Decision makers des Juli 14 überhaupt gewusst und wissen können, welchen Konflikt sie im Begriff waren auszulösen?

Tatsächlich herrschten überall nur vage Vorstellungen von dem, was dann wirklich kam. Der Krieg wurde 1914 überwiegend als „reinigendes Gewitter“, nicht als Katastrophe angesehen. Die verantwortlichen Politiker wie Bethmann Hollweg, Poincaré, Sasonow oder Lloyd George hätten sicher mehr getan, um den Eklat des Juli 1914 zu vermeiden, wenn sie eine einigermaßen präzise Vorstellung von dem gehabt hätten, was dieser Krieg ab August 1914 wurde. Schlachtfelder wie die von Verdun oder der Somme spielten in den Vorstellungen vom Krieg in den Jahren vor 1914 noch keine oder kaum eine Rolle. Solche Visionen waren einigen wenigen klarsichtigen Militärs, Politikern oder Intellektuellen vorbehalten, etwa August Bebel, der 1911 im Reichstag sagte:

„So wird man eben von allen Seiten rüsten und wieder rüsten [...] bis zu dem Punkte, dass der eine oder andere Teil eines Tages sagt: Lieber ein Ende mit Schrecken als ein Schrecken ohne Ende. [...] Dann kommt die Katastrophe. Alsdann wird in Europa der große Generalmarsch geschlagen, auf den hin sechzehn bis achtzehn Millionen Männer, die Männerblüte der verschiedenen Nationen, ausgerüstet mit den besten Mordwerkzeugen, gegeneinander als Feinde ins Feld rücken. [...] Die Götterdämmerung der bürgerlichen Welt ist im Anzuge [...]“(3).

Auch Generalfeldmarschall Helmuth von Moltke d. Ä. hatte 1890 in seiner letzten Rede vor dem Deutschen Reichstag das Bild eines neuen siebenjährigen Krieges beschworen, welcher von Millionenheeren geführt wurde ${ }^{(4)}$. Dabei ging man allerdings

3 Zit. nach: Wolfgang J. Mommsen, „Der Topos vom unvermeidlichen Krieg“, in: Jost DüLfFer und Karl Holl (Hg.), Bereit zum Krieg. Kriegsmentalität im Wilhelminischen Deutschland 1890-1914, Göttingen, 1986, S. 194-224, hier: S. 205.

4 Stig Förster, Helmuth von Moltke, Vom Kabinettskrieg zum Volkskrieg. Eine Werkauswahl, Bonn, 1992, S. 638-641. 
von maximal 1 bis 2 Millionen aus, die die damals gerade entstandenen Heere der allgemeinen Wehrpflicht und des Staatsbürgers in Uniform bereitstellen würden. Von solchen Größenzahlen gingen auch die popularisierenden Darstellungen aus, ebenso die Volkswirtschafts-Experten wie Norman Angell oder - heute berühmter - Ian von Bloch, ein russischer Bankier, der 1899 sein mehrbändiges Werk Der Krieg der Zukunft publizierte $^{(5)}$. Bloch hatte genau errechnet, dass ein langwieriger Krieg die Finanzen aller Nationen zerrütten würde. Die modernen Waffen würden dazu führen, dass sich die Kämpfenden gegenseitig vernichten. „Es wird also das Schlachtfeld der Zukunft mit zahlreichen kleineren und größeren Deckungen wie mit Maulwurfshügeln übersät sein. "Man müsse auch damit rechnen, dass es Heere von mehr als 1 Million Mann geben werde, weshalb die Schlacht der Zukunft bis zu 14 Tage dauern könne (sic!). Blochs Vorstellungen blieben somit - genau wie die all der anderen Kriegskritiker und Propheten des künftigen Krieges - von heute aus gesehen durchaus limitiert, auch wenn sie damals verlacht wurden als realitätsferne bzw. nicht der militärischen Tatsachen kundige Übertreibungen und Spekulationen. Unter den Kritikern zeichnete sich General Friedrich von Bernhardi aus, der im Jahre 1912 zuerst ein zweibändiges Werk über den künftigen Krieg und kurz darauf seine heute noch berüchtigte Schrift Deutschland und der nächste Krieg vorlegte ${ }^{(6)}$. Bernhardi wusste sehr genau, wie man 1-2 Millionen Mann in Bewegung setzen und kontrolliert einsetzen konnte. Er war sich der technischen Schwierigkeiten des Aufmarsches von Millionenheeren klar bewusst und bezog in seine Analyse auch die Möglichkeit ein, dass Kriege ausufern könnten. Aber Bernhardi war, wie mit ihm alle verantwortlichen Militärs der Auffassung, dass man wegen der modernen Steuerungs-, Beobachtungs- und Kommunikationsmöglichkeiten auf jeden Fall diesen Imponderabilien würde gegensteuern können. Erlaubte es das Telefon nicht, eine LKW-Kolonne zielgerichtet an einen Ort zu bringen? Konnte man nicht mit modernen Luftfotografien die Stellungen des Gegners so rekognoszieren, dass sie einem Beschuss nicht lange würden widerstehen können? Wie Bernhardi urteilten eigentlich alle höheren Offiziere, wie eine Artikelserie in der populären Zeitschrift Deutsche Revue im Jahre 1911 zeigte, wo prominente Militärs der europäischen Mächte nach ihren Vorstellungen vom künftigen Krieg befragt wurden und wo sich bei allen individuellen Varianten doch immer wieder dieses Grundmuster der Argumentation zeigte ${ }^{(7)}$. Eine Einstellung, an der auch die Erfahrung der Balkankriege nichts ändern konnte. Im Gegenteil: Angesichts der dort erkannten, fotografierten und beschriebenen Auswirkungen von Schützengräben, Stacheldrahtverhauen und Maschinengewehren, die so stark - für uns - die Kriegserfahrungen des Ersten Weltkrieges andeuteten, kamen die Militärs zu der ebenfalls ganz überwiegenden Überzeugung, dass man solche Hindernisse eben durch noch stärkeren Offensivgeist kompensieren könnte. Der französische Major im Generalstab, de Grandmaison, erklärte sogar in einer 1912 publizierten Instruktionsschrift

5 Johann von Bloch, Die wahrscheinlichen wirtschaftlichen und politischen Folgen eines Krieges zwischen Großmächten, Berlin, 1901.

6 Gerd Krumeich, „Bernhardi et le droit à la guerre“, in: Arndt Weinrich u.a. (Hg.), Guerres futures, guerres imaginées, Paris, 2014.

7 Näheres hierzu mit den Quellenangaben in: Gerd KRUмеich, „Vorstellungen vom Krieg vor 1914“, in: Sönke Neitzel (Hg.), 1900: Zukunftsvisionen der Großmächte, Paderborn u.a., 2002, S. 173-186. 
für Höhere Offiziere, dass die Gefahren des modernen Krieges dazu zwängen, alles offensiv auf eine Karte zu setzen, den Gegner mit riesiger Moral und Enthusiasmus schlicht zu überrennen ${ }^{(8)}$.

Paradigmatisch die 1914 gültigen Grundzüge der oberen Truppenführung aus dem Jahre 1910:

„Der Charakter der heutigen Kriegsführung ist bezeichnet durch das Streben nach großer und schneller Entscheidung. Das Aufgebot aller Wehrfähigen, die Stärke der Heere, die Schwierigkeit, sie zu ernähren, die Kostspieligkeit des bewaffneten Zustandes, die Unterbrechung von Handel und Verkehr, Gewerbe und Ackerbau, dazu die schlagfertige Organisation der Heere und die Leichtigkeit, mit der sie versammelt werden - alles drängt auf rasche Beendigung des Krieges [... $]^{\text {“(9). }}$.

Solches „Expertenwissen“ fand starke Verbreitung. Beispielsweise, so wurde argumentiert, könne man aus dem Maschinengewehr eine hervorragende Angriffswaffe machen. Der ehemalige Generalstabschef Graf Schlieffen beklagte in dem zitieren Artikel von 1911, dass das MG ohnehin kaum von Nutzen sein werde, seien dessen Kugeln doch inzwischen so klein, dass sie die "dicke Haut eines Negers" kaum mehr würden durchschlagen können. Das war zwar sicherlich ein wohlfeiler rassistischer Zynismus, und es ist kaum vorstellbar, dass Schlieffen nicht vom Gegenteil überzeugt war. Zumindest aber erschien ihm eine solche öffentliche Aussage als geeignet, bei den Betroffenen, den Wehrpflichtigen und deren Familien, die Angst vor der militärischen „Feuerprobe“ zu mildern.

Der Krieg, wie er sich im Jahre 1914 präsentierte und im August und September 1914 geführt wurde, entsprach schließlich auch so ziemlich dem, was sich die Kriegsplaner vorgestellt hatten. Im August 1914 hatte die deutsche Armee eine Kriegstärke von ungefähr 2,3 Millionen Mann. Niemals hätte man mit den 13,2 Millionen Soldaten gerechnet, die die deutsche Armee bis Ende 1918 unter den Fahnen hatte.

\section{Vorkriegszeit: Diplomatie und Mentalitäten}

Seit der Agadir-Krise von 1911 lag der Krieg in der Luft und niemand hätte sich gewundert, wenn er bereits Ende 1912 ausgebrochen wäre. Deshalb begannen die Militärs Frankreichs, Englands, Russlands und Deutschlands, ihre Absprachen und Aufmarschpläne zu verfeinern. 1913 kam es zu riesigen Aufrüstungen in diesen Ländern, die wiederum zu nationalistischer Erregung führte ${ }^{(10)}$. Die Franzosen waren

8 Colonel de Grandmaison, Deux conférences faites aux officiers de l'État-Major de l'Armée (février 1911) : La Notion de Sûreté et L'Engagement des Grandes Unités, Paris, 1912. Vgl. insgesamt zur Offensivdoktrin vor 1914: Stephen van EverA, „The Cult of the Offensive and the Origins of the First World War", in: Steven Miller, Sean Lynn-Jones, Ders. (Hg.), Military Strategy and the Origins of the First World War. An International Security Reader, Princeton, 1991, S. 59-108; Jack SNYDER, The Ideology of the Offensive. Military Decision Making and the Disasters of 1914, Ithaka/London, 1984.

9 Zit. nach Jehuda Wallach, Das Dogma der Vernichtungsschlacht. Die Lehren von Clausewitz und Schlieffen und ihre Wirkungen in zwei Weltkriegen, Frankfurt am Main, 1967, S. 119.

10 Gerd Krumeich, Aufrüstung und Innenpolitik in Frankreich vor dem Ersten Weltkrieg, Wiesbaden, 1980; Stig Förster, Der doppelte Militarismus. Die deutsche Kriegsrüstung zwischen Status-quoSicherung und Aggression 1890-1913, Stuttgart, 1985; David Stevenson, Armaments and the Coming of War, Europe 1904-1914, Oxford, 1996. 
überzeugt, dass die Aufrüstung der deutschen Armee den Zweck habe, Frankreich gleichsam ohne Vorbereitung und über Nacht „überfallen“ zu können. In Deutschland wiederum beschwor sogar der gemäßigte Reichskanzler Bethmann Hollweg den nahenden Endkampf zwischen „Slawen und Germanen“.

Unter diesen Voraussetzungen kann es nicht verwundern, dass sich auch der „Volkszorn“ regte. Im April 1913 kam es im grenznahen Nancy zu Ausschreitungen gegen eine Gruppe deutscher Geschäftsreisender, die vom Mob verfolgt wurden. Nur wenige Tage später war die Aufregung wieder groß, als ein deutscher Zeppelin bei Lunéville notlanden musste und die herbeiströmende Menge glaubte, der deutsche Angriff habe begonnen. Beide Zwischenfälle konnten diplomatisch mit gegenseitigen Entschuldigungen bereinigt werden ${ }^{(11)}$. Aber eine wirkliche Beruhigung der Gemüter trat nicht ein. In Frankreich blieb bis zum Ausbruch des Krieges die Vorstellung tief verhaftet, dass Deutschland mit seinen Rüstungen plane, Frankreich unvermutet zu „überfallen“. In Deutschland wiederum machten die französischen Rüstungen und die ständigen Bemühungen Frankreichs um eine demonstrative Konsolidierung der Militärallianz den Eindruck, dass Frankreich nunmehr zielstrebig die „Revanche“ für die Niederlage plane und zusammen mit Russland den „Ring der Einkreisung“ um Deutschland endgültig zuziehen wollte. So kann es nicht verwundern, dass sich in Deutschland nationalistische Aufmärsche, Reden und Feiern häuften. 1913 war ja zudem ein symbolträchtiges Jahr: Wilhelm II. war 25 Jahre als Kaiser im Amt und 100 Jahre zuvor hatte der Aufstand Preußens gegen Napoleon begonnen. Als Höhepunkt der vielen hundert Feiern, Gedenkreden und Aufmärsche wurde im Herbst 1913 das trutzig-germanische Völkerschlachtdenkmal in Leipzig eingeweiht ${ }^{(12)}$.

Gegen Ende des Jahres 1913 nahmen zwar die Spannungen mit Frankreich ab, weil dort eine Regierung mit dem als Deutschlandfreund bekannten Joseph Caillaux ans Ruder gekommen war. Aber die Dauerkonflikte zwischen Deutschland und Russland erreichten nunmehr eine neue Bedrohungsqualität für beide Staaten.

Zunächst rief im November 1913 die Entsendung eines deutschen Generals namens Liman von Sanders in die Türkei, um die dortige Armee zu instruieren, Russlands Protest hervor. Zwar hatte die deutsche Militärmission in der Türkei eine längere Tradition, aber angesichts der aktuellen gegenseitigen Rüstungen und Angstszenarien erschien diese Ernennung im Dezember 1913 der russischen Regierung als direkt bedrohlich. "Sonderkonferenzen“ zwischen Regierung und Generalstab wurden im Februar 1914 einberufen, um zu prüfen, ob und wieweit die russische Armee marschbereit sei. Dabei ging es vor allem um die Fertigstellung besserer Eisenbahn-Linien für den Aufmarsch an der deutschen Grenze, eine finanziell kaum zu bewältigende Aufgabe. Aber Frankreich bot dem russischen Alliierten Hilfe an, denn ein solcher Ausbau des strategisch relevanten Eisenbahnnetzes war für einen kommenden Krieg entscheidend. Im Falle eines Krieges Frankreichs mit Deutschland würde es ausschlaggebend

11 Gilbert Ziebura, Die deutsche Frage in der öffentlichen Meinung Frankreichs von 1911 bis 1914, Stuttgart, 1955; Klaus Wernecke, Der Wille zur Weltgeltung. Außenpolitik und Öffentlichkeit im Kaiserreich am Vorabend des Ersten Weltkrieges, Düsseldorf, 1970.

12 Über diese Feiern ausführlich: Wolfram Sieman,, „Krieg und Frieden in historischen Gedenkfeiern des Jahres 1913“, in: Dieter DüdING u.a. (Hg.), Öffentliche Festkultur. Politische Feste in Deutschland von der Aufklärung bis zum Ersten Weltkrieg, Reinbek, 1988, S. 298-320. 
sein, ob Russland rasch in den Krieg gegen Deutschland eintreten könnte. Die Deutschen erfuhren natürlich von diesen Plänen, und der deutsche Generalstab wurde darüber äußerst nervös, baute doch der deutsche Aufmarschplan von 1905, der sog. „Schlieffen-Plan“, darauf auf, dass man Frankreich besiegen könnte, bevor noch der traditionell schwerfällige russische Aufmarsch an der Ostfront realisiert wäre.

Wie stark die Anspannung und Kriegsfurcht war, mag man an der Tatsache ermessen, dass ein "Alarmruf“ in der regierungsnahen Kölnischen Zeitung vom 2. März 1914 zu einem regelrechten „Pressekrieg“ zwischen Russland und Deutschland führte. In diesem Artikel war von direkten Aggressionsabsichten Russlands die Rede, und vor allem thematisierte er die beherrschende Sorge der deutschen Regierung und Militärs, dass Russland in ungefähr drei Jahren militärisch drückend überlegen sein werde. Eine offensichtlich auf den russischen Kriegsminister Souchomlinow zurückgehende Gegenpolemik in der Petersburger Börsenzeitung heizte die Gemüter weiter an, denn hier hieß es, dass die „Zeit der Beleidigungen vorbei“ sei, und jeder wissen müsse, dass die „russische Armee bald eine aktive Rolle spielen wird“. Zwar erfolgten gegenseitige Dementis, aber die Aufregung in der deutschen und russischen Presse hielt an.

Diese Entwicklung bestätigte natürlich diejenigen in Deutschland, die an den bevorstehenden unvermeidlichen oder gar wünschenswerten Krieg glaubten. Generalstabschef Moltke zeigte sich im Mai 1914 (also lange vor dem Attentat in Sarajewo) voller böser Vorahnungen und drängte immer heftiger auf einen Krieg, solange die deutschen Aussichten auf einen Sieg im Zwei-Fronten Krieg noch realistisch seien. Wie er seinem österreich-ungarischen Kollegen, dem ohnehin auf Krieg (gegen Serbien) drängenden Conrad von Hötzendorff im Mai mitteilte, sei er der Überzeugung, dass „jedes Zuwarten eine Verminderung unserer Chancen bedeutet“. In einem Immediatvortrag beim Kaiser forderte Moltke im Mai 1914 fast ultimativ, dass die deutsche Heeresrüstung endlich so zum Abschluss gebracht werde, dass tatsächlich alle waffenfähigen Deutschen zum Wehrdienst eingezogen würden. In einem Entwurf für dieses Gespräch (dessen genauer Inhalt uns nicht überliefert ist) heisst es: „Nach meinem pflichtgemäßen Ermessen ist es die höchste Zeit, daß wir jeden wehrfähigen deutschen Mann zum Waffendienst ausbilden, soll uns nicht dereinst der vernichtende Vorwurf treffen, nicht alles für die Erhaltung des Deutschen Reiches und der deutschen Rasse getan zu haben.“ Nach einer späteren Aufzeichnung des Staatssekretärs des Auswärtigen Amtes von Jagow, habe Moltke ihm zu jener Zeit in einem persönlichen Gespräch empfohlen, dem für 1916 zu erwartenden Krieg zuvorzukommen und damit die letzte Gelegenheit zu ergreifen, solange Deutschland überhaupt noch Erfolgsaussichten habe.

Der Hamburger Bankier und enge Vertraute des Kaisers, Max Warburg, berichtet über ein Gespräch mit Wilhelm II. am 21. Juni 1914 - 8 Tage vor dem Attentat von Sarajewo -, in dem sich dieser tief beunruhigt über die russischen Rüstungen gezeigt habe und jetzt einen Vorwand zum Losschlagen finden wollte, wenn doch in zwei Jahren alles zu spät sein werde ${ }^{(13)}$. Auch Bethmann Hollweg, der so nachdenkliche und kein wenig „kriegstreiberische“ Reichskanzler, war selber im Frühsommer 1914 von 
starkem Kriegs-Pessimismus befallen, auch wenn er weiterhin einen Präventivkrieg ablehnte ${ }^{(14)}$. Bethmann hatte, genau wie das Auswärtige Amt, große Sorgen wegen kontinuierlich fließender Nachrichten von einem geplanten russisch-britischen Marineabkommen. Dieses war zwar noch in statu nascendi und für sich allein gesehen nicht sonderlich weitreichend ${ }^{(15)}$. Aber man erfuhr alle Verhandlungsschritte direkt durch einen in der russischen Botschaft in London platzierten Spion, den Baltendeutschen Benno von Siebert ${ }^{(16)}$. Diskrete Nachfragen im englischen Außenministerium und vom Auswärtigen Amt initiierte Presseveröffentlichungen insbesondere im Berliner Tageblatt wurden von England mit „diplomatischen“ Ausflüchten und Lügen beantwortet. Die ohnehin so starke „Einkreisungs“-Phobie der deutschen Regierung wurde dadurch selbstverständlich enorm verstärkt.

Entsprechend pessimistisch war die "Gemütslage“ der wichtigsten militärischen und politischen Führer, als die Nachricht vom Attentat von Sarajewo eintraf.

Das Attentat selber war nicht wirklich unerwartet gekommen. Seit der Annexion von Bosnien und der Herzegowina im Jahre 1908 war der Irredentismus in der dortigen serbisch-stämmigen Bevölkerung enorm gewachsen. Aktivistische (Klein)-gruppen brüteten Revolutions- und Attentatspläne aus, wobei sie von mehreren serbischen Geheimorganisationen mit Logistik und Waffen unterstützt wurden. Dabei handelte es sich u.a. um die „Schwarze Hand“ und die Narodna Obrana („Volksschutz“). Wichtig ist, dass diese Geheimbünde wiederum mit radikalen Offizieren des serbischen Militärs verknüpft waren - nicht aber mit der Regierung ${ }^{(17)}$.

Anlass für die Visite des Thronfolgers in Bosnien waren Truppenmanöver. Die Gruppe der jungen bosnischen Verschwörer kannte den Weg des Korsos genau, und nicht weniger als sechs Attentäter hatten sich an der Strecke postiert. Einer der Attentäter, Newdeljko Cabrinovic, warf am Appelkai einen Sprengsatz auf das offene Cabriolet, in welchem sich der Thronfolger und seine Gemahlin befanden. Er traf aber nur den nachfolgenden Wagen, unter dem die Sprengladung explodierte. Trotz des Attentats wurde die Fahrt fortgesetzt, man verfuhr sich sogar, der Wagen musste zurücksetzen, und just an dieser Ecke stand der Student Gavrilo Princip, der die Gelegenheit erkannte, seinen Revolver zog und aus nächster Nähe den Erzherzog und seine Gemahlin erschoss.

Das Attentat rief überall in Europa Entsetzen hervor, und die Mächte waren sich im Grunde einig, dass Österreich-Ungarn von Serbien Genugtuung erhalten sollte. Aber die Wiener Regierung kam sehr bald zu dem Entschluss, den „Königsmord“ zu nutzen, um ein für allemal diesen unruhigen und gefährlichen Nachbarn zu entmachten.

14 Hierzu nach wie vor am besten: Konrad Jarausch, The Enigmatic Chancellor. Bethmann Hollweg and the Hubris of Imperial Germany, New Haven/London, 1973.

15 Vgl. auch C. Clark, Die Schlafwandler (Anm. 1), S. 418ff., der zeigt, dass es den Briten vor allem darauf ankam, Russlands Ambitionen zu begrenzen.

16 Benno von Siebert (Hg.), Diplomatische Aktenstücke zur Geschichte der Ententepolitik der Vorkriegsjahre, 2 Bde., Berlin, 1925; vgl. für die Korrespondenz im Frühjahr 1914 ebd., Bd. 2, S. 804ff.

17 C. Clark, Die Schlafwandler (Anm. 1), der eine sehr genaue und farbige Darstellung des Attentats gibt, nimmt Kenntnis und Verstrickung auch der serbischen Regierung an, hat dies aber m. E. nicht hinreichend belegen können; vgl. nach wie vor: Vladimir Dedijer, Die Zeitbombe. Sarajewo 1914, Wien, 1967. 
Man steuerte auf einen Krieg mit Serbien zu. Und der Ministerrat der österreichischungarischen Monarchie beschloss am 7. Juli, der serbischen Regierung unakzeptable Kompensations-Bedingungen zu stellen, damit man endlich Krieg gegen Serbien führen könne ${ }^{(18)}$. Zu dieser politischen Strategie wurde Wien durch die deutsche Regierung ermutigt, welche sich sehr schnell auf den Standpunkt stellte, dass der Mord von Sarajewo genutzt werden solle, mit den Serben abzurechnen. Am 5. 7. ging man einen entscheidenden Schritt weiter. In Übereinstimmung mit der politischen und militärischen Führung des Reiches erklärte Kaiser Wilhelm II. dem österreichischen Sonderbeauftragten Graf Hoyos, dass Deutschland die Doppelmonarchie in diesem Konflikt auch dann militärisch unterstützen werde, wenn Russland für Serbien Partei ergreife - auch auf die Gefahr eines Großen Krieges hin. Das deutsche Kalkül in der Krise war ebenso elaboriert wie für die anderen Mächte undurchsichtig. Man entschied, die Krise zu nutzen um herauszufinden, ob Russland wirklich kriegsbereit sei. Sollte Russland im Falle eines Krieges Österreich-Ungarns mit Serbien für das kleine slawische „Brudervolk“ eingreifen, so sei es besser, den Krieg, der ohnehin drohte, besser jetzt zu führen als etwas in zwei Jahren, wenn Russland das Deutsche Reich überrüstet haben werde. Deshalb forderte man die anderen Großmächte auf, tatenlos zu sehen, wie Österreich-Ungarn mit Serbien nach Belieben umsprang. Sollte Russland nicht, wie gefordert, beiseite stehen und den unter seiner traditionellen Protektion stehenden kleinen slawischen Staat gegen Österreich-Ungarn unterstützen, würde dies den Kriegswillen Russlands ad oculos zeigen. Dann aber wolle man „lieber jetzt als später“ Krieg gegen Russland führen. Die deutsche Regierung umschrieb während der gesamten Krise ihre Forderung, dass die anderen Mächte nicht eingreifen dürften, als Bemühen um eine „Lokalisierung“ des Konfliktes. Damit wollte man den Anschein erwecken, dass es darum ginge, den „Brandherd“ einzugrenzen. Aber angesichts der Tatsache, dass spätestens seit dem Wiener Kongress von 1815 europäische Großmachtpolitik darin bestanden hatte, „lokale“ Konflikte durch gemeinsame Verhandlungen zu de-eskalieren, waren die anderen Mächte von dieser Forderung Deutschland verblüfft. Warum sollte man nicht auch, wie man es trotz aller Spannungen zwischen den beiden großen Bündnissystemen noch 1913 im Falle Albaniens geschafft hatte, verhandeln und gemeinsam eine Lösung finden? Wollte Deutschland etwa unbedingt Krieg?

Dem Zarenreich war es vollständig unmöglich, angesichts der Empörung der eigenen Öffentlichkeit den Gewaltakt an der kleinen slawischen Brudernation hinzunehmen. Die russischen Militärs drängten auf eine Mobilmachung gegen Österreich-Ungarn. Die zutiefst aufgebrachte russische Regierung begann nun ebenfalls, einen großen Krieg ins Auge zu fassen, obwohl der Zar genauso unschlüssig blieb wie sein Vetter Wilhelm II. Mobilmachungen Russlands, in welcher konkreten Form auch immer, waren aber das non plus ultra des Bedrohungs-Szenariums, das die deutschen Militärs seit Jahren aufgebaut hatten und das die Politiker inzwischen insgesamt teilten: Sie wussten, dass der Erfolg des deutschen Kriegsplanes, des „Schlieffenplans“, davon abhing, dass Russland erst zu einem Zeitpunkt in den Krieg eingreifen konnte, wenn

18 Der Beschluss des Ministerrates der österreichisch-ungarischen Monarchie vom 7.7.1914, abgedr. bei G. Krumeich, Juli 1914 (Anm. 13), S. 243; C. Clark, Die Schlafwandler (Anm. 1), ignoriert diesen Beschluss, einen Krieg mit Serbien führen zu wollen. 
Deutschland Frankreich schon nahezu besiegt hatte. Jeder Tag, den die Russen eher mit ihrem Aufmarsch fertig waren, bedeutete deshalb eine einschneidende Schwächung dieses sakrosankten Planes.

Alles spitzte sich damit auf die Frage zu, wer als erster die Mobilmachung befehlen würde. Dies war am 30. Juli eindeutig Russland, welches lange zwischen verschiedenen Formen von Teilmobilmachung geschwankt hatte, aber jetzt dem kategorischen Wunsch der Generale nachgab, nicht länger zu zögern - auch die Russen wussten natürlich um die Nachteile einer späten Mobilmachung im erwarteten „kurzen Krieg“. Damit war das deutsche Bemühen erfolgreich, Russland als Schuldigen am Ausbruch des Weltkrieges anprangern zu können.

Wer war schuld? Zweifellos hat die unverantwortliche Erpressungs- und Bluffpolitik der deutschen Regierung den größten Anteil an der Entfesselung des Krieges. Aber nicht allein die Deutschen waren schuld an den bis ins Unerträgliche gesteigerten Spannungen der Vorkriegszeit. In der so intensiven Kriegsschuld-Debatte nach dem Ersten Weltkrieg hat der große französische Historiker und Pädagoge Jules Isaac abschließend folgendermaßen geurteilt: Der Krieg sei gekommen, weil für keine der beteiligten Nationen der Frieden das höchste Gut gewesen sei. Hinter dieser lakonischen Feststellung verbirgt sich die tiefe Einsicht, dass den Politikern von 1914 trotz mancher Vorahnungen ein europäischer Krieg als durchaus noch machbar erschien. Alle Mächte glaubten, dass der Krieg nach wie vor nach Clausewitz’ Formel die „Fortsetzung der Politik mit anderen Mitteln“ sei. Es gab ja auch noch keine Ächtung des Krieges, wie sie erst 1928 im sog. Briand-Kellog-Pakt erfolgte. 1914 war ein Angriffskrieg als echte Alternative beim Scheitern politischer Verhandlungen, wie es die Bismarckzeit so häufig gesehen hatte, ein Bestandteil des europäischen ius publicum! Und zudem hatte niemand für einen mehr als drei Monate dauernden Krieg geplant, und alle glaubten wirklich, Weihnachten wieder zu Hause zu sein.

Hätte irgendeiner der Staatsmänner, die 1914 Europa in den Krieg führten, so gehandelt wie er es tat, wenn er gewusst hätte, was die Somme und Verdun 1916 an Tod, Verstümmelung und Vernichtung von ganzen Landschaften bringen würden? Man hatte vom Gaskrieg keine Vorstellung, genauso wenig wie von flächendeckender schwerer Artillerie, von Tanks oder Bomben werfenden Flugzeugen. Der Krieg, wie er 1914 begann, hatte mit dem von 1916 bis 1918 wenig zu tun. Auch das hatte Clausewitz 100 Jahre zuvor vorausgesehen, als er urteilte, dass Krieg immer die Tendenz habe, sich hin zum „absoluten“ Krieg zu erweitern, wenn die Politik ihm keine Zügel anlege. Die Wahrheit dieser Analyse zeigte sich dann besonders stark im Zeitalter der Millionenheere und der ungeheuren industriellen Produktivität der europäischen Gesellschaften auch für den Großen Krieg.

\section{Vom europäischen Krieg zu Europas Krieg in der ganzen Welt}

Worin aber bestanden eigentlich die charakteristischen und von Clausewitz sogenannten „Weiterungen“ des Krieges in den Jahren zwischen 1914 und 1918?

Wir können in dieser Hinsicht fünf maßgebliche Faktoren nennen, deren Abfolge bzw. Rangordnung aber wohl nicht präzisiert werden kann:

a. Propaganda und Hass

b. Der Krieg der großen Massen 
c. Industrialisierung des Krieges

d. Die „Mondialisierung“ des Krieges am Beispiel der Dardanellen

e. Das Nicht-mehr-Aufhören-Können

\section{a. Propaganda und Hass}

Das Wort Propaganda hat erst im Ersten Weltkrieg seine heutige Bedeutung angenommen. Vor 1914 war es noch synonym mit Produktwerbung, man machte also „Propaganda" für Schuhcreme oder Cognac ${ }^{(19)}$. Begonnen hat die überall einsetzende Kriegspropaganda mit den Anklagen der Alliierten gegen die „Gräueltaten“ der deutschen Armee beim Einfall in Belgien. Die tatsächlich mit unglaublicher Brutalität durchgeführten Strafaktionen der Deutschen gegen meist nur vermeintliche „Heckenschützen“ oder „franc-tireurs“(20) lösten nicht nur starken Protest aus, sondern massive Beschuldigungen des zum barbarischen Ungeheuer mutierten „Boche“. Dieser wurde mit riesigem Bild- und Wortaufwand aller denkbaren Verbrechen geziehen. Brandschatzung, Raub, Vergewaltigung, Verstümmelung, auch von Frauen und Kindern, fanden sich täglich in der illustrierten Massenpresse Frankreichs, Englands und der USA. Dies war umso bedeutender, als bebilderte Tageszeitungen damals ein noch ganz neues und deshalb Aufsehen erregendes Medium waren: Erst ab 1911 war es mit dem neuen Rotationsdruck möglich geworden, die Tageszeitungen mit Fotos zu illustrieren - und entsprechend glaubwürdig erschien diese Bild-Propaganda. So ist etwa über die Beschießung der Kathedrale von Reims oder die Inbrandsetzung der Bibliothek von Löwen - also zwei Kleinodien der europäischen Kultur - mit großem Aufwand an Fotos berichtet worden. Man spürt heute noch das ungläubige Entsetzen, welches sich der Betroffenen bei diesen abgrundtiefen und der deutschen Brutalität zugeschriebenen Kriegsverbrechen bemächtigte. Wichtig ist, dass diese Gräuelpropaganda anfangs weniger von staatlichen Stellen geleitet wurde, sondern aus privater Initiative von Zeitungsmachern und Intellektuellen - Künstlern, Lehrern und Professoren - ausging. Das machte ihre Intensität und Mobilisierungskraft aus. Denn die deutschen Armeen standen ja tatsächlich in Frankreich, und es ging wirklich darum, alle Geister auf die nationale Verteidigung einzustimmen ${ }^{(21)}$.

Für England lagen die Dinge etwas anders, da das Land nicht wirklich vom Einmarsch des Feindes bedroht war. Aber im Unterschied zum wilhelminischen Deutschland war die englische Presse schon seit Jahrzehnten frei von jeder Zensur gewesen. So war man in oft exzessiv betriebenem politischen Streit geübt und brauchte diese polemischen Fähigkeiten nur noch auf den deutschen „Hunnen“ zu übertragen, was mit großem Erfolg geschah.

Die Deutschen hatten dieser geistigen Mobilmachung wenig entgegenzusetzen, und sie beherrschten auch bis zum Kriegsende nicht die Prinzipien der modernen

19 Näher hierzu mit der Spezialliteratur: Gerhard Hirschfeld, Gerd Krumeich, Deutschland im Ersten Weltkrieg, Frankfurt, 2013, Kap. 5.

20 John Horne und Alan Kramer, Deutsche Kriegsgreuel 1914. Die umstrittene Wahrheit, Hamburg, 2004.

21 Gerd Krumeich, „Ernest Lavisse und die Kritik an der deutschen ,Kultur‘, 1914-1918“, in: Wolfgang J. Mommsen (Hg.), Kultur und Krieg. Die Rolle der Intellektuellen, Künstler und Schriftsteller im Ersten Weltkrieg, München, 1996, S. 143-154. 
Kriegspropaganda, nämlich die noch so absurdeste Behauptung vielgestaltig, massenhaft und bunt immer wieder aufs Neue zu verbreiten. Während des ganzen Krieges blieben sie propagandistisch weitgehend in der Defensive: „Sind wir die Barbaren?“ so lautete der Text eines der bekanntesten Propagandaplakate. Oder man versuchte zu zeigen, dass keinesfalls nur die Deutschen die Kulturstätten der anderen Nationen in Brand schossen, sondern dass die Franzosen und Engländer das selber taten (was ja zum Teil zutreffend war) ${ }^{(22)}$. Bei den zur Kriegsfinanzierung sehr wichtigen Anleiheplakaten hatten die Deutschen zwar hübsche und teilweise künstlerisch sogar avantgardistische Bilder anzubieten, aber von den Gräueln des Gegners war hier nichts zu spüren, im Unterschied etwa zu französischen und englischen Plakaten, wo ohne weiteres eine Vergewaltigung durch den Hunnen oder ein ans Kreuz geschlagener alliierter Soldat die Stimmung anheizen und das Anleihegeld locker machen sollte und konnte.

\section{b. Der Krieg der großen Massen}

Genaue Zahlen lassen sich bei der weltumspannenden Ausdehnung des Ersten Weltkriegs und angesichts der damaligen Kommunikations- und Zählungs-Möglichkeiten naturgemäß nicht nennen. Die Historiker gehen von einer Gesamtzahl von ca. 70 Millionen im Laufe des Krieges mobilisierten Männern aus. In den ersten zwei Jahren strömten 2 Millionen Freiwillige vor allem auf der Seite der Alliierten zu den Waffen, ab 1916 herrschte überall ein Wehrpflicht-System. Das Deutsche Reich mobilisierte zwischen 1914 und 1918 insgesamt 13,3 Millionen Männer, Österreich-Ungarn 9 Millionen. Dazu kamen die Türkei mit 1,6 Millionen und Bulgarien mit 1,2 Millionen. Somit standen auf Seiten der Mittelmächte insgesamt wohl 25 Millionen Mann im Felde ${ }^{(23)}$.

Für die Alliierten und assoziierten Mächte sind folgende Zahlen relativ gesichert: Russland mobilisierte mehr als 15 Millionen, Frankreich (einschließlich Kolonien) 8,2 Millionen; Großbritannien (inklusive Dominions und Indien) 9,5 Millionen, Italien beteiligte sich mit 5,2 Millionen, Belgien mit ca. 1/2 Million, Rumänien und Serbien mit jeweils 1 Million und schließlich die USA mit 3,9 Millionen Soldaten. Der Beitrag der übrigen ca. 20 Staaten wird auf ungefähr 1 Million geschätzt. Insgesamt wurden also auf Seiten der Alliierten ca. 45 Millionen Mann unter die Fahnen gestellt.

Was die Verlustzahlen im Krieg angeht, so sind diese heute immer noch nicht gut gesichert, die Gesamtzahlen an Toten und Verwundeten schwanken zwischen 20 und 35 Millionen Mann.

Bei einer Gesamtzahl von 70 Millionen Soldaten sind mindestens 10 Millionen, wahrscheinlich wohl eher 12 bis 13 Millionen, getötet worden: Man darf von 15-20 Millionen Verwundeten ausgehen, allein Deutschland hatte 4,5 Millionen mehr oder weniger schwer Verwundete! Und die Türkei hatte um die 325.000 Tote, wobei

22 Christina Котт, Préserver l'art de l'ennemi? Le patrimoine artistique en Belgique et en France occupées, 1914-1918, Bruxelles, 2006.

23 Reichsarchiv (Hg.), Der Weltkrieg 1914 bis 1918, Kriegsrüstung und Kriegswirtschaft, Anlageband, Berlin, 1930, Tabellen 19ff. über die militärischen Kräfteverhältnisse vor und im Krieg. 
nach anderen Quellen noch 500.000 durch Erkrankung gestorbene Soldaten hinzukamen, und das bei insgesamt 3 Millionen im Krieg eingesetzten Männern ${ }^{(24)}$.

Das waren ungeheure Zahlen, die wohl auch heute noch der Hauptgrund dafür sind, dass von diesem Krieg in vielen Ländern als der „Große Krieg“ gesprochen wird.

Auch die Kriegsgefangenen-Zahlen erreichten Dimensionen, die die Menschheit zuvor nicht einmal geahnt hatte: Seriöse Berechnungen liegen zwischen 7 und 8 Millionen Gefangenen, das sind mehr als 10\% aller mobilisierten Soldaten! Nach Statistiken aus der Zwischenkriegszeit waren Ende 1918328.000 Soldaten in britischer, 350.000 in französischer und 916.000 Soldaten in österreich-ungarischer Gefangenschaft. Allein 2,25 Millionen waren in russischer Gefangenschaft und das Deutsche Reich hatte kurz vor Ende des Krieges insgesamt 2,4 Millionen Soldaten aus 134 Staaten in Gefangenschaft.

Kriegsgefangenschaft wurde im Ersten Weltkrieg also zu einem Massenphänomen. Und Kriegsgefangene in Millionenzahl bedeuteten eine bislang noch nie gekannte Belastung der Kriegsgesellschaften. Man „kompensierte“ diese durch Arbeitseinsatz, der bisweilen auch die Fesseln der Haager Konventionen sprengte, nämlich die Vorschrift, dass Kriegsgefangene nicht zur Arbeit für Kriegszwecke eingesetzt werden dürfen. Kriegsgefangene waren auch vielfachen politisch motivierten Repressalien ausgesetzt, und besonders die russischen Kriegsgefangenen hatten unter rassistischen Vorurteilen zu leiden, allerdings fallen oft angestellte Vergleiche mit der Misshandlung und Ausrottungspolitik im 2. Weltkrieg schwer, denn die Unterschiede sind insgesamt zu stark gewesen. Im Ersten Weltkrieg war der kriegsgefangene Soldat durchgehend noch Mitmensch und kein Abfall ${ }^{(25)}$ !

Im Unterschied zum Zweiten Weltkrieg war der Erste Weltkrieg kein Krieg gegen die Zivilbevölkerung und deshalb auch noch kein wirklich „totaler“ Krieg. Es gibt allerdings eine Ausnahme, nämlich das Schicksal der armenischen Minderheit in der Türkei. Die „armenische Frage“ hatte die Großmächte schon in den 1870er Jahren beschäftigt. Ab 1910 stand sie im Mittelpunkt der „jungtürkischen“ Bewegung, die eine Vorherrschaft des Islam über andere Religionen und Ethnien zustande bringen wollte. Im Krieg wurde dieses Projekt auf grausame Weise realisiert. Erste Niederlagen der Türkei gegen russische Truppen wurden auf das Konto von „antitürkischen Elementen“ verbucht, es begannen Hinrichtungen und Vertreibungen größeren Ausmaßes. Das geschah unter den Augen deutscher Generäle, die im Rahmen des Bündnisses in der Türkei tätig waren. Diese unterstützten das Vorhaben der Türken, die „unzuverlässige“ armenische Bevölkerung aus den kriegsnahen Gebieten zu deportieren. Auch die deutsche Regierung bekundete ihr Desinteresse am Schicksal der Armenier. Ihr ging es allein darum, den türkischen Bundesgenossen „bis zum Ende des Krieges

24 Zahlen-Quellen vor allem bei Rüdiger Overmans, „Kriegsverluste“, in: Gerhard Hirschfeld u.a. (Hg.), Enzyklopädie Erster Weltkrieg, 3. Aufl., 2009; vgl. auch: Michael Clodfelter, Warfare and Armed Conflicts. A Statistical Reference to Casualties and other Figures, London, 2002.

25 Vgl. bes.: Uta Hinz, Gefangen im Großen Krieg, Essen, 2005; Jochen Oltmer (Hg.), Kriegsgefangene im Europa des Ersten Weltkriegs, Paderborn, 2006; Christian Westerhoff, Zwangsarbeit im Ersten Weltkrieg, Paderborn, 2012; Heather Jones, Violence against Prisoners of War in the First World War, Britain, France and Germany 1914-1920, Cambridge, 2013. 
an unserer Seite zu haben, gleichgültig, ob darüber Armenier zu Grunde gehen oder nicht", so Reichskanzler Bethmann Hollweg in einem Kommentar zu einem diplomatischen Bericht vom Dezember 1915.

Die Massaker begannen am 24. April 1915, als in Konstantinopel nahezu 2.500 armenische Intellektuelle verhaftet und ermordet wurden. Das war der Beginn eines regelrechten Völkermordes. Türkische Truppen fuhren fort, die armenischen Minderheiten in den verschiedenen Regionen der Türkei systematisch auszurotten. Entweder wurden sie direkt ermordet, etwa durch Ertränken ganzer „Schiffsladungen“ von Menschen auf dem Meer, oder sie wurden in Scheunen zusammengepfercht und verbrannt. Am stärksten aber wird die Deportation mit regelrechten Todesmärschen in die Wüste erinnert. Die wichtigsten Vernichtungsorte der deportierten Armenier lagen in Syrien entlang des Euphrats in der Wüste, „wo die Opfer zumeist verhungerten, wenn nicht bestellte Mörder ganze Deportiertenzüge umbrachten“ (W. Gust). In der Hauptsache waren die Todesmärsche Ende 1916 beendet, aber es kam noch bis Kriegsende zu weiteren Verfolgungen und Mordaktionen. Die Zahlen des Völkermordes, der bis heute von der türkischen Regierung bestritten wird, dessen Leugnung aber von europäischen Staaten wie etwa Frankreich unter Strafe gestellt worden ist, liegen zwischen 1 Million und 1,5 Millionen. Bekannt geworden sind die Armeniergräuel insbesondere durch den deutschen Weltenbummler und Kriegsjournalisten Armin T. Wegner, dessen Fotoreportagen und Vorträge nach 1919 die Öffentlichkeit bewegten und die trotz einiger Verfälschungen noch heute die wichtigste Dokumentation dieses ersten Völkermordes des 20. Jahrhunderts sind.

\section{c. Industrialisierung des Krieges}

Schon die gegebenen Zahlen zeigen, wie der Krieg geradezu Tag um Tag an „Masse“ gewann, wie er zur immer schneller arbeitenden Tötungs- und UnterdrückungsMaschine für Millionen Menschen wurde. Dies ging natürlich nur durch die massenmäßige Herstellung von Waffen aller Art. Auch hier geschah etwas, wovon man vorher keine Ahnung gehabt hatte, nämlich die Fähigkeit der industrialisierten Gesellschaften, in kürzester Zeit wesentliche Teile ihrer Produktion auf den Krieg umzustellen.

Ein Beispiel ${ }^{(26)}$ : Während noch zu Kriegsbeginn die Gewehre einzeln manufakturmäßig gefertigt und zusammengesetzt wurden, wurde ab Oktober 1914 (als man erkannte, dass der Krieg länger dauern würde), auf Massenproduktion umgestellt, wobei alle Arten von feinmechanischen Fabriken genutzt wurden, z.B. Fahrrad-, Schreibmaschinen- und Nähmaschinen-Fabriken, die jetzt anfingen, Einzelteile von Gewehren herzustellen. Insgesamt waren 100 Fabriken mit der Herstellung der einzelnen Gewehrteile beschäftigt. So kam man in Deutschland ab März 1915 zu einer Massenfertigung von 250.000 Gewehren pro Monat! Und in den anderen Ländern lief die Gewehr- und Geschützproduktion mit zumindest gleich starker industrieller Effizienz ab. Während und durch den Krieg ist das Fließbandverfahren, welches Henry Ford ab 1907 zum ersten Mal praktiziert hatte und das vor dem Krieg von den europäischen Manufakturen noch sehr kritisch angesehen wurde, zum Standard der neuen Industrialisierung geworden.

26 Näheres hierzu bei Hirschfeld/Krumeich, Deutschland im Ersten Weltkrieg, Kap. 8: Die Industrialisierung des Krieges, mit den zeitgenössischen Quellen (Anm. 19). 
Die ständig steigernden Anforderungen von der Front ließen die Waffenproduktion binnen kurzem in zuvor unvorstellbare Größenordnungen anwachsen. Nach einer Zusammenstellung aus den 1930er Jahren wurden für die deutsche Artillerie im letzten Jahre des Krieges pro Monat 11 Millionen Geschosse für ca. 100 verschiedene Typen von Gewehren und Kanonen hergestellt. Die Monatsfertigung von Patronen soll bereits im März 1916220 Millionen betragen haben. Eine ähnliche Entwicklung gilt auch für die Maschinengewehre. In den ersten Monaten nach der Mobilmachung wurden ca. 200 Maschinengewehre pro Monat gefertigt, im Jahre 1915 wurde diese Quote vervierfacht und 1916 gelangte man zu einer Monatsproduktion von 2300 MGs.

Ein weiteres und berühmtes Beispiel dieser Industrialisierung der Waffenproduktion sind die Tanks:

Die Engländer hatten schon im September 1915 einen ersten Prototyp fertiggestellt, der den Spitznamen „Little Willie“ trug. Weil dieses Gefährt in der Tat aussah wie ein großer Wassertank, nannte man es fortan „Tank“. Im Februar 1916 ging der erste Tank (Mark I) in Serienproduktion. Von diesem Ungetüm gab es zwei Varianten: eine mit zwei kleinen Geschützen vom Kaliber 5,7 und eine andere mit fünf Maschinengewehren. Beide Tanks wogen 28 t. Der Kanonen-Panzer sollte der Infanterie den Weg durch das feindliche Stellungssystem bahnen, während der MG-Tank direkt in den Infanterie-Kampf eingreifen konnte. Im Laufe der Zeit wurden vier Serien dieser Tanks eingesetzt (Mark I-IV). Daneben wurde noch ein leichterer Tank angefertigt, der nur $14 \mathrm{t}$ wog und mit 4 MGs ausgerüstet war. Bei einem Infanterie-Angriff sollte dieses leichte Gefährt den Durchbruch in die Tiefe ermöglichen. Die Soldaten konnten in Halbdeckung hinter diesem wendigen Gerät vorwärts stürmen. Im Laufe des Krieges haben die Briten nahezu 2.000 schwere und leichte Tanks gebaut.

In Frankreich begann die Produktion verschiedener Tank-Modelle ebenfalls bereits Mitte 1916. Als man dann mit der fehlgeschlagenen Nivelle-Offensive und den Soldatenstreiks im Frühjahr 1917 erkannte, dass ein weiteres „Ausbluten“' der französischen Armee unter allen Umständen zu verhindern war, wurde der neuen Tankwaffe viel Aufmerksamkeit gewidmet. Ein Volltreffer wurde der ab 1917 in Auftrag gegebene Kampfpanzer Renault M 17, mit einem Gewicht von nur 6,7 t und einer leichten, gut schwenkbaren Kanone vom Kaliber 3,7. Er war sehr geländegängig, gut gepanzert und wurde in großen Stückzahlen gebaut, nicht weniger als 3.200 Exemplare bis Kriegsende.

Dagegen hatte Deutschland die Entwicklung der Tankwaffe regelrecht verschlafen. Die Oberste Heeresleitung unterschätzte die Wirkung der Tanks auf unverantwortliche Weise. Noch nach dem Krieg hat Ludendorff in seinen Memoiren behauptet, dass Tanks ja in Wirklichkeit auch nur wirksam sein könnten, wenn die „Moral“ der Truppe ohnehin bereits gebrochen sei!

Ähnlich dramatische Steigerungen und Erneuerungen, die hier aber nicht im Einzelnen geschildert werden sollen, waren das Flugzeug und die Geschütze ${ }^{(27)}$.

Vielleicht am zukunftsweisenden war die Erfindung der Gaswaffe. Schon vor dem Krieg waren Reizgase gelegentlich Mittel der Kriegführung gewesen und deshalb auch von den Haager Konventionen für fortgesetzten Gebrauch verboten worden. Die 
Pattsituation an der Westfront aber führte dazu, dass sich die deutsche Heeresführung im Frühjahr 1915 entschloss, Chlorgas gegen die feindlichen Linien einzusetzen, um den Krieg wieder „,in Bewegung“ zu bringen. Das war die Geburtsstunde von modernen Massenvernichtungsmitteln. Am 17. April 1915 setzte das Oberkommando vor Ypern das von dem deutsche Chemiker Haber entwickelt Chlorgas ein. Das Ergebnis waren ca. 1.200 tote und 3.000 verwundete (erblindete) französische Soldaten. Stärker als diese direkten Verluste war die „moralische“ Wirkung des Gaseinsatzes. Er verursachte eine Massenpanik unter den französischen Fronttruppen, die aber ohne weitreichende Folgen für den Positionskrieg blieb. Doch die Deutschen hatten angefangen, und die Empörung hierüber ist bis heute nicht verklungen. Allerdings waren die anderen Mächte sehr schnell ihrerseits bereit, sich dieses bislang verpönten Kriegsmittels zu bedienen. Im Herbst 1915 eröffneten die Briten die erste alliierte Gas-Offensive bei Loos in Belgien. Experten haben gezählt, dass bis 1918 insgesamt 408 Operationen mit flüssigen Gasen (Chlor und Phosgen) erfolgten. Als Höhepunkt der Kampfgas-Entwicklung gilt die als "Lost“ bezeichnete chemische Substanz, die deutsche Experten im Juli 1917 bei Ypern einsetzten. Das war eine nicht nur über die Atemwege, sondern über die gesamte Haut einwirkende Substanz, gegen die es keinen Schutz gab. Ein einziger Angriff vor Ypern (13. Juli 1917) hatte ca. 15.000 Gasverletzte - aber nur relativ wenig Tote - zur Folge. Besonders wirksam und verbreitet wurden die schon bei Verdun und dann an der Somme eingesetzten Gasgranaten, die in speziell hierfür entwickelten Gasgranatenwerfern in die feindlichen Stellungen geschossen wurden. Im Juli 1916 wurden solche Granatwerfer in der Somme Schlacht zum ersten Mal massiv auf britischer Seite eingesetzt. Und im März 1918 verfügte die britische Armee über mehr als 200.000 solcher Geschütze. Die anderen Nationen konstruierten ähnlich „erfolgreiche“ Gaswaffen. Insgesamt wurden im Weltkrieg von allen Kriegsparteien ca. 112.000 t Gas eingesetzt, wobei Deutschland fast die Hälfte dieser Menge produzierte. Allerdings blieb der Einsatz auf den Frontbereich beschränkt. Gas erweckte zwar stets Entsetzen und Paniken, führte aber im Ersten Weltkrieg zu relativ wenig Todesopfern. Nach zuverlässigen Schätzungen sind nur ca. 3,5\% der Kriegsopfer auf Gaseinwirkung zurückzuführen (ca. 500.000 Verletzte und 20.000 Tote).

Alle Mächte sorgten auch in einer Art „stillem Übereinkommen“ dafür, dass Gas nicht gegen die Zivilbevölkerung und auch nicht aus Kampfflugzeugen eingesetzt wurde. Irgendwie scheute man sich noch, den Krieg in dieser Richtung total werden zu lassen ${ }^{(28)}$.

\section{d. Die „Mondialisierung“ des Krieges am Beispiel der Dardanellen}

Während die Deutschen 1915 auf dem Balkan selbst aktiv in die Kämpfe eingriffen, beschränkten sie sich im Osmanischen Reich zunächst auf umfangreiche Militärhilfe sowie die Ausrüstung und Ausbildung der türkischen Truppen. Zu diesem Zweck wurden die Aufgaben der bereits lange vor dem Krieg etablierten deutschen Militärmission erheblich erweitert. Deren Leiter, der preußische General Liman von

28 Zum Gaskrieg insgesamt: Olivier LePICK, La Grande Guerre chimique 1914-1918, Paris, 1998; Dieter Martinetz, Der Gaskrieg 1914-1918, Bonn, 1996; Wolfgang Zecha, „Unter die Masken“. Giftgas auf den Kriegsschauplätzen Österreich-Ungarns im Ersten Weltkrieg, Wien, 2000; vgl. auch: M. SzöLLösIJANZE, Fritz Haber 1868-1934. Eine Biographie, München, 1998. 
Sanders, war seit 1913 als Marschall und Generalinspekteur des Osmanischen Heeres mit der kompletten Reorganisation ihrer Armeen betraut; außerdem erhielt er nach dem Kriegseintritt des Osmanischen Reiches im November 1914 den Oberbefehl über die 1. sowie im März 1915 zusätzlich über die 5. Türkische (Gallipoli) Armee. In dieser Funktion kommandierte Liman von Sanders die erfolgreiche Abwehr der zahlenmäßig überlegenen französischen und britischen Invasionstruppen, darunter auch starke australische und neuseeländische (ANZAC) Einheiten, bei deren Invasion Ende April 1915 auf der Halbinsel Gallipoli an der Dardanellen-Küste. Zu Recht haben einige Historiker die ungemein verlustreichen Kämpfe bei den Landeversuchen der Alliierten auf Gallipoli, wo Felsen und Steinstrand kaum ausreichende Deckung ermöglichten, mit dem blutigen Stellungskrieg entlang der Westfront (seit November 1914) sowie in den Alpen (seit Mai 1915) verglichen. Die angreifenden Alliierten verloren etwa 180.000 Mann, die Verluste der verteidigenden Türken waren sogar doppelt so hoch. In der historischen Erinnerung der beteiligten Nationen, vor allem der Briten und ihrer ehemaligen Dominions, spielt „Gallipoli“ bis heute eine außerordentlich bedeutsame Rolle. Dabei wird von der „westlich“ orientierten Geschichtsschreibung trotz aller „transnationalen“ Bekenntnisse oft „übersehen“, wie heldenhaft der türkische Abwehrkampf bei Gallipoli war und wie sehr die erfolgreiche Abwehr dieser Aggression die türkische Nation mit Stolz erfüllt hat. „Gallipoli“ war der Beginn des Aufstiegs von Kemal Pascha, der die Verteidigung dort mit größter Energie und strategischer Klugheit organisiert hatte.

\section{e. Das Nicht-mehr-Aufhören-Können}

Die totale Schlacht kannte keine Pause mehr, keine Unterbrechung, um etwa die Verwundeten zu bergen. Der Grund dafür sind moralische, technische und wirtschaftliche Faktoren:

Viele Millionen Soldaten genauso wie eine neue und bis dahin nicht bekannte Ausrichtung der gesamten Ökonomie auf den Krieg waren notwendig. Um das zu erreichen, wurden aber auch die Emotionen immer stärker beansprucht. Die Propaganda redete vom Heiligen Krieg und vom Überlebenskampf gegen barbarische Feinde. Einen heiligen Krieg kann man aber erst beenden, wenn das satanische Übel ausgerottet ist.

Genauso bedeutend für die Permanenz des Krieges war, dass die Überbeanspruchung der Ökonomien zu einer gigantischen Staatsverschuldung führte, denn Kriegsausgaben wurden zum größten Teil mittels Anleihen finanziert, die man bei der eigenen Bevölkerung aufnahm oder aber von auswärtigen Kapitalgebern erhielt. Der große ökonomische Krieg geschah also „auf Pump“ und unter der Voraussetzung, dass nach einem siegreichen Abschluss der Gegner die Zeche zu zahlen hätte (wie man es ja auch 1870 gemacht hatte). Dies war in allen kriegführenden Ländern das offizielle Programm und Alternativen waren nicht vorgesehen. Der von Friedensfreunden immer wieder erhobene und auch von einigen offiziellen Stimmen propagierte „Frieden ohne Annexionen und Kontributionen“ war also ökonomisch überhaupt nicht machbar. Von daher führte die Totalisierung des Krieges zu einer immer geringer werdenden Bereitschaft, über Friedensmöglichkeiten auch nur nachzudenken. Dem totalen Krieg musste ein totaler Sieg folgen, sonst war auch der Sieger verloren. Unter diesem Umständen wurde der Krieg im Laufe des Jahres 1918 von denjenigen Mächten 
abgebrochen, die moralisch und wirtschaftlich tatsächlich „am Ende“ waren, und das waren die multinationalen Großreiche: Russland, Österreich-Ungarn, das Osmanische Reich und das Deutsche Reich in seiner alten Form.

\section{Versailles und die Folgen ${ }^{(29)}$}

Zur Friedenskonferenz, die am 18. Januar 1919 begann, waren Repräsentanten von 27 Nationen eingeladen. Die Verlierer des Weltkriegs hatte man nicht eingeladen ein Novum in der Geschichte der Friedensverträge. Jetzt kam es nur noch darauf an, die unterschiedlichen Positionen und Forderungen auf Seiten der Alliierten zu harmonisieren. Während die USA nach wie vor eine Art „Wilson-Frieden“ anstrebten, also einen Friedensschluss gemäß den 14 Punkten des amerikanischen Präsidenten, bestanden die Franzosen auf einem echten Siegfrieden, in dem Deutschland für alle Schäden, die das ausgeblutete Frankreich erlitten hatte, einschließlich seiner immensen Kriegsschulden, aufkommen sollte. Zudem verlangte die französische Regierung eine territoriale Neuordnung, die einen Angriff von seiten Deutschlands auf alle Zeiten unmöglich machen sollte. Die Briten argumentierten in dieser Hinsicht zweifellos moderater: Großbritannien war nicht daran interessiert, Frankreich in einem neu zu gestaltenden Europa eine dominierende Rolle zuzubilligen. Einigkeit bestand unter den Alliierten lediglich darin, dass Deutschland künftig keine weltpolitische Rolle mehr einnehmen durfte, was nach damaliger Ansicht hieß, auf Kolonien, ein starkes Heer und eine Kriegsmarine zu verzichten.

Symptomatisch für die Grundstimmung unter den Vertretern der in Versailles versammelten Mächte war die Eröffnungsrede des französischen Staatspräsidenten Poincaré am 18. Januar 1919. Nicht von ungefähr hatten die Franzosen exakt diesen Tag und diesen Ort gewählt, an dem im Jahre 1871 das Deutsche Reich gegründet worden war. Poincaré sprach von einem verbrecherischen Krieg der Deutschen und von der „blutbefleckten Wahrheit“, die inzwischen auch aus „deutschen Archiven entwichen“ sei. Damit spielte der Präsident auf Veröffentlichungen an, die der bayerische Revolutionsführer Kurt Eisner wenige Tage zuvor aus den Münchner Archiven zutage gefördert hatte und die beweisen sollten, dass das kaiserliche Deutschland den Weltkrieg mutwillig vom Zaun gebrochen hatte.

Wilson hegte allerdings starke Vorbehalte gegenüber der vor allem von den Franzosen geforderten radikalen Abrechnung mit dem besiegten Deutschland. Amerikaner und Briten teilten die Befürchtung, dass ein gedemütigtes und auf Dauer schlecht behandeltes Deutschland kommunistisch werden könne. Während die angelsächsischen Mächte zu Kompromissen bereit waren, um die deutsche Republik als kontinentales Bollwerk gegen einen von Sowjetrussland ausgehenden „Weltkommunismus“ aufzubauen, bestand Frankreich mit aller Schärfe auf einer vollständigen réparation aller Kriegsschäden.

29 Einen präzisen knappen Überblick vermittelt: Eberhard KolB, Der Frieden von Versailles, München, 2005; die wichtigsten Quellen finden sich bei: Klaus Scнwaвe (Hg.), Quellen zum Friedensschluss von Versailles, Darmstadt, 1997. Zur Auseinandersetzung um Versailles und zu den Auswirkungen: Gerd Krumeich (Hg.), Versailles 1919. Ziele - Wirkung - Wahrnehmung, Essen, 2001; Manfred BoemeKe u.a (Hg.), The Treaty of Versailles: A Reassessment after Seventy Years, Cambridge, 1998. 
Tatsächlich war ja der materielle Schaden, den Frankreich erlitten hatte, immens: Zwei Millionen Hektar Ackerland, Wald und bewohnte Flächen waren vernichtet; mehr als 300.000 Häuser entweder vollständig zerstört oder schwer beschädigt; 60.000 Kilometer Straßen und mehr als 5.000 Kilometer Eisenbahnlinien mussten wiederhergestellt werden. Zudem war Frankreich bei seinen Verbündeten mit insgesamt sieben Milliarden Dollar verschuldet.

Erst Mitte April 1919 wurde eine deutsche Vertretung zur Friedenskonferenz in Versailles zugelassen. Die Übergabe des alliierten Entwurfs der Friedensbedingungen an die Deutschen fand in Anwesenheit der Repräsentanten von 27 Siegerstaaten im Hotel „Trianon Palace“ in einer dramatisch aufgeladenen Stimmung statt. Clemenceaus kurze Ansprache an die deutsche Delegation war ein Meisterwerk an kalter Verachtung: „Sie haben vor sich die Bevollmächtigten der kleinen und großen Mächte, die sich versammelt haben, um den fürchterlichsten Krieg auszufechten, der ihnen aufgezwungen worden ist. Die Stunde der Abrechnung ist da.“

Die Härte der Friedensbedingungen stieß in Deutschland auf ein vielstimmiges Echo aus Ungläubigkeit, Verzweiflung, Protest und kategorischer Ablehnung. Vor allem die umfangreichen Gebietsabtretungen im Westen und Osten - Deutschland sollte unter anderem Elsass-Lothringen an Frankreich sowie die Provinzen Posen und Oberschlesien und den größten Teil Westpreußens an Polen abtreten - sowie der Verlust aller deutschen Kolonien erschienen als inakzeptabel. Als geradezu ungeheuerlich aber wurde der Artikel 231 des Vertragswerks empfunden, mit dem eine juristische Haftung des Deutschen Reiches für alle Kriegsschäden „verursacht durch die Aggression Deutschlands und seiner Verbündeten“ festgestellt werden sollte. Dieser „Kriegsschuldartikel“, der den Anspruch der Alliierten auf umfangreiche Wiedergutmachungs-Leistungen (Reparationen) begründete, entwickelte sich zu einem moralischen Verdikt und damit zu einem „deutschen Trauma“ (Eberhard Kolb), das die gesamte Weimarer Zeit überschattete.

Der Vertrag von Versailles war der Auftakt für den Abschluss weiterer Friedensabkommen, welche die Alliierten in den folgenden 13 Monaten mit Deutschlands Verbündeten schlossen: mit Österreich (10.9.1919 in St. Germain), mit Bulgarien (27.11.1919 in Neuilly), mit Ungarn (4.6.1920 in Trianon) sowie mit der Türkei (10.8.1920 in Trianon). Sämtliche Pariser „Vorortverträge“ folgten dem gleichen Muster: Die Verliererstaaten mussten umfangreiche Gebietsabtretungen akzeptieren, ihre Streitkräfte erheblich reduzieren und beträchtliche Reparationen zusagen. Vor allem jene Staaten, die territoriale Einbußen erlitten, betrieben in der Folgezeit eine Politik des Revisionismus mit dem erklärten Ziel, die ihnen aufgezwungenen Bestimmungen außer Kraft zu setzen oder sogar rückgängig zu machen.

\section{Ein „30-jähriger Krieg“"? ${ }^{(30)}$}

Nach den Erfahrungen zweier Weltkriege ist immer wieder gefragt worden, ob nicht der Zweite Weltkrieg eine direkte Konsequenz des Großen Krieges von 1914-1918

30 Für Einzelnachweise verweise ich hier besonders auf die soeben erschienenen 3 Bände von: The Cambridge History of the First World War, hg. von Jay WInTER and The Editorial Committee of the International Research Centre of the Historial de la Grande Guerre, Cambridge UP, 2014. Verwiesen sei besonders auf: Robert Gerwarth, The continuum of violence (ebd., Bd. 2, S. 638-662); besonders 
gewesen ist. Der französische Sozialwissenschaftler Raymond Aron hat dies ebenso betont wie Hanna Arendt in ihren Elementen und Ursprüngen totaler Herrschaft. Der englische Sozialhistoriker Eric Hobsbawn nannte sein Buch über diese Epoche Das Zeitalter der Extreme.

Bereits während des Zweiten Weltkrieges war die Kontinuitätslinie zum Ersten Weltkrieg für viele eine Selbstverständlichkeit. Hitlers Tischgespräche zeigen dies genauso wie Churchills Erinnerungen. Auch de Gaulle betonte schon 1941 in einer Rundfunkansprache, dass „die Welt einen dreißigjährigen Krieg gegen die Herrschaft Deutschlands" führe.

Unter diesem Aspekt wurde die Zeit zwischen den beiden Weltkriegen tatsächlich zu einer „Zwischenkriegszeit“.

Für die These vom „30-jährigen Krieg“ spricht zunächst, dass der Vertrag von Versailles keinen echten Frieden schaffen konnte. In der Folgezeit kam es immer wieder zu kriegerischen Auseinandersetzungen im Rahmen des neuen Staatensystems von möglichst ethnisch „reinen“ Nationalstaaten, was zu ständigen Ausgrenzungen und Vertreibungen führte. Weiterhin bereiteten sich die Staaten auf einen in kurzer Frist erwarteten Folgekrieg vor. Die Franzosen versuchten, mit der Maginot-Linie eine Art „chinesische Mauer“ um ihr Land zu ziehen. Überall wurden „Gasschutz“-Maßnahmen eingeführt, die Panzer- und Flugzeugindustrie boomte. Mindestens so wichtig aber war, dass in Deutschland ein sehr starker Revanchismus herrschte. Das Trauma des so „schandvoll“ verlorenen Krieges wollte nicht weichen und verband sich dann in der ökonomischen Krise ab 1928 zu einem virulenten Protestpotential, aus dem der Nationalsozialismus seine spezifische Dynamik erhielt. Hitlers Charisma beruhte in erster Linie darauf, dass er versprach, das Unrecht von Versailles zu tilgen. Seine innen- und außenpolitischen Erfolge nach 1933 schienen ihm Recht zu geben - der Sieg über Frankreich im Jahre 1940 wurde von den meisten Deutschen als siegreiches Ende des Ersten Weltkrieges interpretiert.

Für die Auffassung, es habe einen 30-jährigen Krieg gegeben, wird auch die Kontinuität der Vernichtungspolitik angeführt. Zwischen der Ausrottung der Armenier 1915 und dem Völkermord an den Juden bestehe eine direkte ideologische und „technische“ Kontinuität.

Zweifellos hat es hier ein „Lernen“ gegeben. Allerdings sind die Armenier-Gräuel doch örtlich sehr begrenzt und ein im Ersten Weltkrieg einzigartiges Ereignis geblieben, sie gehören also nicht strukturell zum „Vernichtungskrieg“, wie dies ab 1941 der Fall war.

Gegen die These vom 30-jährigen Krieg spricht weiterhin die Tatsache, dass „Versailles" keineswegs nur eine Abrechnung der Sieger mit den Verlierern war, sondern dass hier auch ein starker Wille zur Schaffung eines neuen und friedlichen Europa herrschte, zusammen mit ernsthaften Bemühungen um eine Weltfriedensordnung, symbolisiert im „Völkerbund“.

ertragreich für die Mächteverschiebungen und die Bevölkerungs-Umschichtungen nach dem Krieg: Peter Gatrell, „War after the War: Conflicts 1919-1923“, in: John Horne (Hg.), A Companion to World War I, Wiley-Blackwell, 2010, S. 558-575. 
Die These vom 30-jährigen Krieg aber ist deshalb so griffig und offensichtlich realitätsnah, weil kaum ein Zeitgenosse daran zweifelte, dass „Versailles“ und die folgenden wirtschaftlichen Probleme - vor allem die Inflation - ursächlich für Hitlers Aufstieg zur Macht waren.Die notwendige historische Differenzierung zwischen beiden Weltkriegen darf diese zeitgenössische Überzeugung nicht außer Acht lassen. Tatsächlich konnte ja erst nach zwei Weltkriegen im Laufe von nur 30 Jahren Europa ein Kontinent des Friedens werden.

\section{Résumé}

Cet article essaie de donner une idée des dimensions et de l'ampleur qu'eut la Grande Guerre. Il présente d'abord l'état d'esprit et les opinions des gouvernements et des responsables militaires avant 1914. On y voit naître la certitude grandissante d'une guerre inéluctable et imminente. Il y eut des psychoses de guerre et la profonde conviction que les autres puissances tramaient des complots, si bien qu'en août 1914, tout le monde, de tous côtés, était convaincu de mener une guerre juste, pour la défense de son pays. On prépara la guerre dans l'idée qu'elle ne durerait au grand maximum que jusqu'à Noël. L'article montre ensuite comment et pourquoi la guerre a dégénéré en guerre de masses et en guerre mondiale: une totalisation qui n'est pas achevée en 1918 mais qui est sans doute un "progrès" notable par rapport à tout ce que l'humanité avait vu jusqu'alors. La Deuxième Guerre mondiale n'est pas pour autant une suite "logique » de la Grande Guerre: il faudra des facteurs nouveaux pour en faire une vraie guerre totale. 